\title{
Application of Dynamic State and Parameter Estimation Techniques on Real-World Data
}

\author{
Lingling Fan, Senior Member, IEEE, Zhixin Miao, Senior Member, IEEE, Yasser Wehbe, Student Member, IEEE
}

\begin{abstract}
The objective of this paper is to apply two dynamic state and parameter estimation techniques to real-world data. Dynamic state and parameter estimation can estimate equivalent generator internal voltages and rotor angles (dynamic states) during electromechanical dynamics. In addition, parameters such as the transient reactances and inertia can be estimated. A set of real world data recorded by Eastern Interconnection Phasor Measurement Units (PMU) regarding a generator trip event is analyzed in this paper. An estimation algorithm developed by Chow et al in [1] was applied to estimate the states and parameters of a transfer path. An alternative estimation method based on the classic generator model and swing equation is presented in this paper and applied to the real world PMU data with the aid of digital filtering technology. Challenges in real-world data applications are identified in this paper.
\end{abstract}

Index Terms-Dynamic State Estimation, Dynamic Parameter Estimation, Phasor Measurement Units, Interarea Oscillations

\section{INTRODUCTION}

$\mathbf{P}$ Hasor Measurement Units (PMU) data in Eastern Interconnection (EI) collected by Real Time Dynamics Monitoring System (RTDMS) database [2] has a $30 \mathrm{~Hz}$ sampling rate. This is a much faster sampling rate compared with the conventional state estimation with a $0.2 \mathrm{~Hz}$ sampling rate. The conventional state estimation estimates bus voltages and phase angles every five minutes and deals with power flow problems. With a higher sampling rate, dynamic state estimation concerning low frequency electromechanical dynamic $(<3 \mathrm{~Hz})$ becomes feasible.

Dynamic state and parameter estimation has been applied in synchronous generator parameter and state estimation using digital fault recording (DFR) data [3]-[6]. The machine parameters to be estimated include the resistance and inductances of the armature winding, field resistance, magnetizing inductances and the effect of saturation. The states to be estimated include damper winding currents [4]. Digital fault recording data has a very high sampling rate (many samples per cycle) and can capture electromagnectic transients. These transients are sufficient for estimation of machine electric circuit parameters.

The difference between the machine circuit parameter estimation and the estimation investigated in this paper resides in both objectives and available data. While DFR data can capture electromagnetic transients, PMU data cannot capture

This work was supported in part by the Department of Energy Smart Grid Investment Grant under Award Number DE-OE0000369. L. Fan, Z. Miao and Y. Wehbe are with the Department of Electrical Engineering, University of South Florida, Tampa, FL 33620 (Emails: linglingfan@usf.edu, zmiao@usf.edu, ywehbe@gmail.com ). electromagnetic transients. Instead, PMU data can capture electromechanical dynamics, hence parameters related to electromechanical dynamics such as inertia $(H)$ and damping factor $(D)$ can be estimated. In addition, dynamic states such as generator rotor angle and internal voltage can also be estimated. The available data from DFR are stator phase currents and voltages at the terminals of the machine, and the field voltage and current. The available data from PMUs are voltages, voltage phase angles, real and reactive power.

Observers have been used to estimate dynamic states in [7]. A single-machine infinite-bus system model is used to design a nonlinear observer. The states (rotor angle and rotor speed) are estimated using a nonlinear observer. Kalman filter can be considered as a type of observers. Feasibility of using PMU data for dynamic state estimation applying Extended Kalman Filter (EKF) technology is discussed in [8]. [8] finds that 30 $\mathrm{Hz}$ PMU data sampling rate is sufficient to estimate dynamic states. In [9], the EKF problem is formulated to include parameters as extended states. Such formulation makes both dynamic state and parameter estimation possible. Parameters such as inertia $H$, damping factor $D$ and transient reactance $x_{d}^{\prime}$ are estimated in [9]. In [10], parameters (inertia and damping factor) in a multi-machine power system are estimated using the EKF technique. The EKF technique estimates the states at current time step based on the measurements and the state estimation at the previous time step. Sensitivity studies carried out in [8] show that noise and disturbance type affect convergence and estimation accuracy.

Kalman filter technique is suitable for real-time estimation and requires adequate computational power. In off-line studies, alternative methods have been proposed to avoid using estimation from the previous steps. This can in turn save computing resources. An estimation method relying on measurements only is developed by Chow et al in [1]. The estimation method is based on a two-generator system model capturing electromechanical oscillatory dynamics. Two PMUs are assumed to be installed at the two ends of a transfer path. Bus voltage magnitudes, phase angles and the through current magnitude and angle are assumed to be known. The algorithm tackles reactance estimation first and then generator internal voltage and rotor angle estimation. Using these estimations along with the observation of the oscillatory frequency, an aggregate machine inertia is computed. Individual inertia for each area is then computed. The algorithm is based on small signal analysis of electromechanical dynamics. Based on the analysis, two principles are developed: (i) bus voltage magnitude oscillations are proportional to the rotor angle deviation and dependent on line and equipment impedances, and (ii) 
the oscillation frequency is dependent on the system inertia. Based on these two principles, the estimation algorithm was developed. Adaption of this method has been developed in [11] to include a shunt capacitive branch.

The second estimation is based on the classical generator model and the swing equation. Detailed description, case studies and sensitivity analysis of the method are presented in [12]. Since the method deals with finite differences, it is called as the "finite difference method".

The two methods, Chow's method and the finite difference method, will be applied in a set of real-world PMU data. The data represents a generator tripping event occurred at a transfer path. The transfer path was subject to interarea oscillations during the transient period.

The major contributions include two elements:

1) Challenges in applying Chows method [1] into identifying system parameters from real PMU data are tackled. The challenges are: (1) identification of a radial path from multiple PMU data based on voltage magnitude deviations and (2) obtaining rotor speed deviations in off-nominal frequency scenarios.

2) Finite difference based least square estimation method is applied using real world PMU data for system identification. Digital filter technique is applied on the noise polluted finite difference data to obtain meaningful results.

The remainder of the paper is organized as follows. Section II describes the two estimation methods. Section III presents the event data. Section IV describes the real-world data analysis procedure and the challenges. Section $\mathrm{V}$ concludes the paper.

\section{Estimation Methods}

\section{A. Chow's Method}

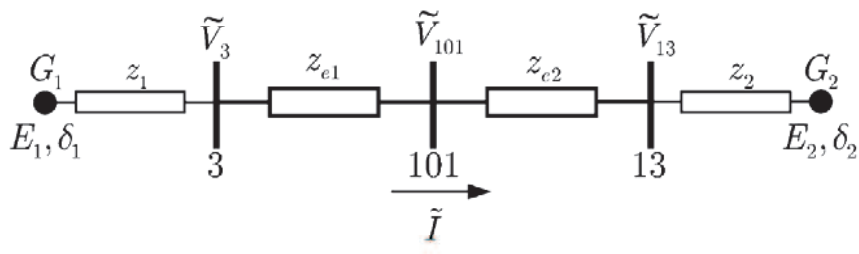

Fig. 1. Two-area system considered in [1].

A two-area system is shown in Fig. 1. This area is equivalent to a single-machine infinite-bus system shown in Fig. 2. The relationship of the inertia of the equivalent machine $(H)$ and the two machines $\left(H_{1}, H_{2}\right)$ is as follows: $H=\frac{H_{1} H_{2}}{H_{1}+H_{2}}$.

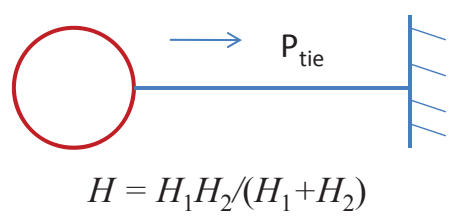

Fig. 2. The equivalent single-machine infinite-bus system.
For the single-machine infinite-bus system, it is assumed that the machine is represented by a constant voltage source behind a reactance. The dynamics equations of the system are expressed in Fig. 3. Linearizing the equations leads to a linear system expression $\dot{X}=A X$ where $X$ is the vector of the state variables $\left(X=[\Delta \delta, \Delta \omega]^{T}, \delta\right.$ being the rotor angle and $\omega$ being the rotor speed). The expression of the system matrix $A$ is also shown in Fig. 3. A pair of complex eigenvalues of the system matrix determines the electromechanical oscillation mode. The frequency of the oscillation mode is determined by the imaginary part of the eigenvalues and it is shown Fig. 3. It is noted that the oscillatory frequency is related to the inertia constant. The greater the inertia is, the lower the oscillatory frequency. If the oscillatory frequency can be observed or computed from the event data, it will be possible to compute the aggregated inertia constant $H$.

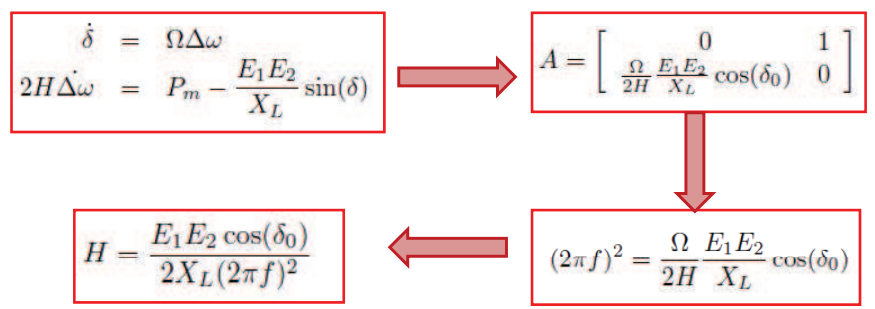

Fig. 3. Computing aggregated inertia.

In order to obtain the aggregated inertia, the required data include the generator internal voltages $E_{1}$ and $E_{2}$, the total reactance including the two transient reactances, the oscillatory frequency and the steady-state rotor angle difference of Area 1 and Area 2. The oscillatory frequency can be observed or computed by Prony analysis. The steady-state rotor angle difference can be computed from the steady state power transfer level if $E_{1}$ and $E_{2}$ are known. $E_{1}, E_{2}, \delta_{1}$ and $\delta_{2}$ can be found given the terminal voltage phasor and current phasor if the transient reactances are known. Hence the foremost important step is to estimate the reactances: the external one $X_{e}$ and the transient reactances $x_{d 1}^{\prime}$ and $x_{d 2}^{\prime}$.

Chow et al [1] developed the relationship of the line impedances and the voltage magnitude deviation at three buses on a radial line (the sending bus, the receiving bus and the middle point bus) as shown in (1) and (2). The middle point voltage phasor is computed from the two terminal voltage phasors. Impedances $Z_{e 1}$ and $Z_{e 2}$ are assumed equal. From there, the reactances can be computed. All impedances in Fig. 1 are assumed to be pure reactances. This assumption is reasonable for transmission lines where resistances are much smaller compared to reactances.

For the radial path, the voltage magnitude deviation at a bus $\Delta V(x)$ is proportional to the deviation of the deviation of the rotor angle difference $(\Delta \delta)$ according to the linearized swing equation and the linearized voltage magnitude expression [1]:

$$
\Delta V(x)=f(x) \Delta \delta .
$$

where $\Delta V$ is the voltage magnitude deviation, $x$ relates to location and $\Delta \delta$ is the rotor angle deviation. This rotor angle represents the rotor angle difference between the two 
generators at the terminals. Hence it is apparent that the at all locations on the transfer path, the voltage deviation is proportional to the rotor angle deviation. If the rotor angle increases, the voltages also increase. If the rotor angle decreases, the voltages also decrease.

In general, we can observe the swing pattern of voltage magnitudes and pick the buses that swing in phase. These buses are located in the same transfer path.

The voltage magnitude deviations at different locations will be related to each other based on the following equations [1]:

$$
\begin{aligned}
V_{3 n}\left(1-a_{101}\right) a_{101} & =V_{101 n}\left(1-a_{3}\right) a_{3} \\
V_{13 n}\left(1-a_{101}\right) a_{101} & =V_{101 n}\left(1-a_{13}\right) a_{13}
\end{aligned}
$$

where:

$$
\begin{aligned}
a_{3} & =\frac{x_{d 2}^{\prime}+x}{x_{d 1}^{\prime}+x+x_{d 2}^{\prime}}, \\
a_{13} & =\frac{x_{d 2}^{\prime}}{x_{d 1}^{\prime}+x+x_{d 2}^{\prime}}, \\
a_{101} & =\frac{x_{d 2}^{\prime}+x / 2}{x_{d 1}^{\prime}+x+x_{d 2}^{\prime}},
\end{aligned}
$$

and $V_{j n}$ is the normalized voltage magnitude oscillation. Buses 3, 13 and 101 are the sending bus, receiving bus and middle point bus as shown in Fig. 1.

From the above equations, transient reactances can be estimated. In turn, the internal voltage and rotor angle will be computed based on circuit analysis. With $E_{1}, E_{2}$ and the total reactance available, the aggregated inertia constant $H$ will be computed based on the equation presented in Fig. 3.

The next step is to find $H_{1}$ and $H_{2}$ of the two areas. Momentum conservation is assumed in [1] for the system and hence the sum of the change of momentum is zero:

$$
H_{1} \Delta \omega_{1}+H_{2} \Delta \omega_{2}=0 \text {. }
$$

Equation (3) can be written as

$$
\frac{H_{1}}{H_{2}}=-\frac{\Delta \omega_{2}}{\Delta \omega_{1}} .
$$

Given the aggregated inertia constant and estimated speed deviation, individual area's inertia can be found.

The detailed procedure of application will be demonstrated in Section IV.

\section{B. Finite Difference Method}

The finite difference method assumes that a PMU is installed at the terminal of a generator. The classic generator model is assumed. The internal voltage phasor $E \angle \delta$ has a relationship with the terminal voltage $V \angle \theta$ and current phasor $I \angle \alpha$ as follows:

$$
E \angle \delta=j x_{d}^{\prime} I \angle \alpha+V \angle \theta .
$$

In (5), there is an unknown parameter $x_{d}^{\prime}$ and an unknown state $E$. $E$ is assumed to be constant in classical generator model. The assumption is valid when the automatic voltage regulator is not considered. Equation (5) can further be simplified by removing the dynamic variable $\delta$ :

$$
E^{2}=V^{2}+x_{d}^{\prime 2} I^{2}+2 V I x_{d}^{\prime} \sin (\theta-\alpha) .
$$

In order to find the values where $E$ is almost constant and $x_{d}^{\prime}$ to be constant, Equation (6) will be fitted to a constant value while tuning $x_{d}^{\prime}$. This curve-fitting technique can be accomplished by the MATLAB function lsqcurvefit. The output will be a value for $x_{d}^{\prime}$ achieving the least variance for $E$. With $x_{d}^{\prime}$ known, $E$ and $\delta$ can be computed through (5).

The inertia $H$ of the machine can be found through the swing equation:

$$
\frac{2 H}{\Omega} \frac{\partial^{2} \delta}{\partial t^{2}}=P_{m}-P_{e}
$$

where $P_{m}$ is the mechanical power delivered to the machine through the turbine and $P_{e}$ is the electrical power delivered to the stator. $P_{e}$ can be computed given the terminal voltage and current phasor measurements:

$$
P_{e}=\operatorname{Re}\left(V I^{*}\right)
$$

In (7), $\frac{\partial^{2} \delta}{\partial t^{2}}$ can be calculated numerically since $\delta$ is available at all time steps. On the other hand, $P_{m}$ is constant within the study time and we can assume that $\frac{d P_{m}}{d t}=0$. Hence

$$
\frac{2 H}{\Omega} \frac{\partial^{3} \delta}{\partial t^{3}}=-\frac{\partial}{\partial t}\left(P_{e}\right)
$$

which leads to

$$
H=-\frac{\Omega}{2}\left(\frac{\partial P_{e}}{\partial t}\right) /\left(\frac{\partial^{3} \delta}{\partial t^{3}}\right) .
$$

In (10), the only unknown parameter $H$ should be constant and can be computed from the derivatives of electric power and rotor angle.

Using finite difference to find time derivatives has been seen in the literature. For example, in [13], the derivatives of frequency are computed through finite difference. In generator parameter estimation [4], [6], the derivatives of currents are computed using finite difference.

\section{A REAL-WORLD DYNAMIC EVENT}

The RTDMS data set for an event of generator trip is used for dynamic state and parameter estimation. North American Synchro Phasor Initiative (NASPI) RTDMS shows significant oscillations. Frequency plots are shown in Fig. 4.

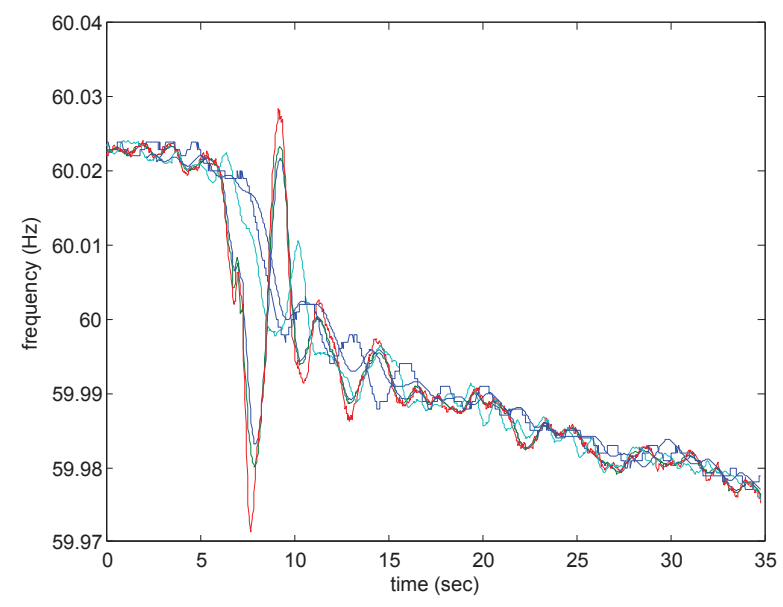

Fig. 4. Frequency plots. 
Seven locations are selected and will be called $P_{0}-P_{6}$ according to the respective position from the west to the east of the EI region. The voltage phase angles are plotted in Fig. 5 and the voltage magnitudes are shown in Fig. 6. The voltage phase angles are relative angles. The reference bus is chosen to be a bus located in Tennessee Valley Authority (TVA).

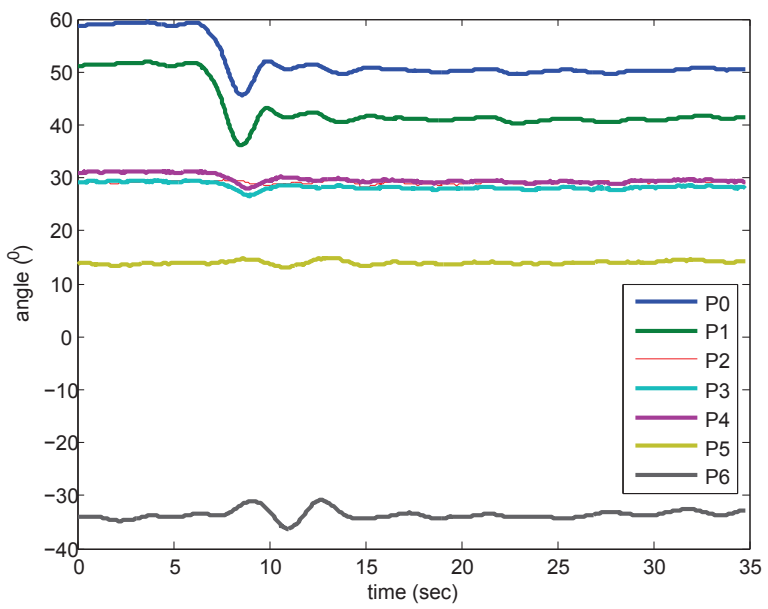

Fig. 5. Voltage phase angles. Reference bus is located in TVA.

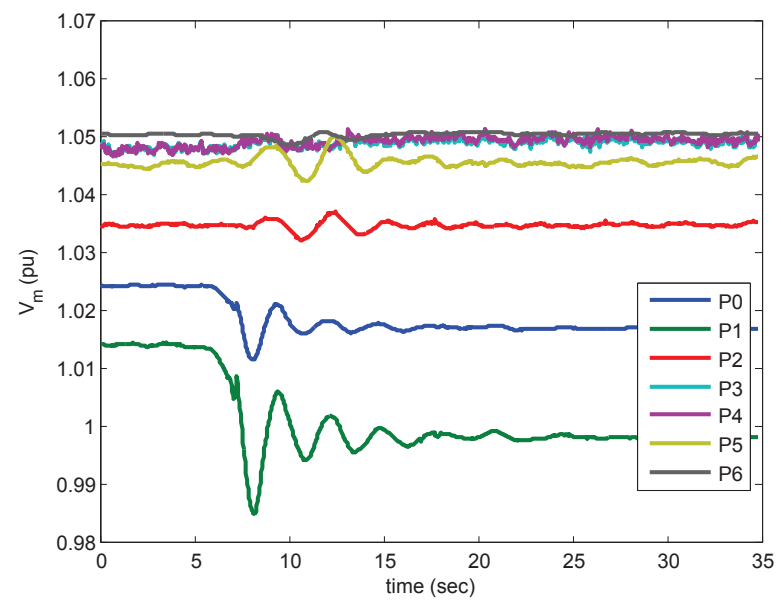

Fig. 6. Voltage magnitudes.

From Fig. 5, we can observe that $\angle \bar{V}_{0}>\angle \bar{V}_{1}>\ldots>\angle \bar{V}_{6}$. Hence the power transfer direction is from west to east since the voltage phase angles decrease from west to east. This can also be confirmed by the power flow measurements on some PMUs. It is noted that only few PMUs have data for real power and reactive power, though the majority of the PMUs have voltage phasor data. The frequencies measured at these locations decreased below $60 \mathrm{~Hz}$ which show that load in the system exceeds generation.

Starting from $t=8 s$, it is obvious that the system suffers an active power unbalance since the frequencies decrease. Voltage phase angles of $P_{0}$ and $P_{1}$ have significant reduction (about 10 degree) while the voltage phase angles of the other locations $\left(P_{2}-P_{6}\right)$ have insignificant reduction. The power transfer from the west to the east showed a reduction after the event.
To apply Chow's method, a radial transfer path should be identified. In the real systems, these PMUs are not located along a radial path. Hence it is necessary to identify two locations which have a connection path being the most similar to a radial path. For two buses located on a radial path, it has been proved in [1] that the oscillation trends of the bus magnitudes should agree with each other. Observing the voltage magnitudes in Fig. 6, we find that the two buses $P_{0}$ and $P_{1}$ have similar oscillation trends. $P_{2}$ and $P_{5}$ have similar oscillation trends.

In the following section, Chow's method will be applied to the PMU data from $P_{0}$ and $P_{1}$. Finite difference method will be applied to the PMU data from $P_{0}$. Chow's method was also applied to the PMU data from $P_{0}$ and $P_{2}$. From Figs. 5 and $6, P_{0}$ and $P_{2}$ can be found having phase angles and voltage magnitudes swinging against each other. $P_{0}$ and $P_{2}$ can represent two coherent areas. This set of data however leads to negative inertia in one area which makes no sense. This is due to the violation of radial path assumption if $P_{0}$ and $P_{2}$ are chosen.

\section{Data Analysis Procedure And Results}

\section{A. Chow's method}

Data from the two PMU installed at $P_{0}$ and $P_{1}$ is used. A fictitious midpoint is assumed. The voltage phase angle and magnitude at $P_{0}(345 \mathrm{kV})$ are shown in Fig. 7 and power flow on one major line from $P_{3}$ to $P_{4}$ is shown in Fig. 8. Since there are no power flow data recorded in the PMU installed in $P_{0}$ and $P_{1}$, power flow data on the line $P_{3}$ to $P_{4}$ is used to compute the line through current. The line flow is about 500 MW.
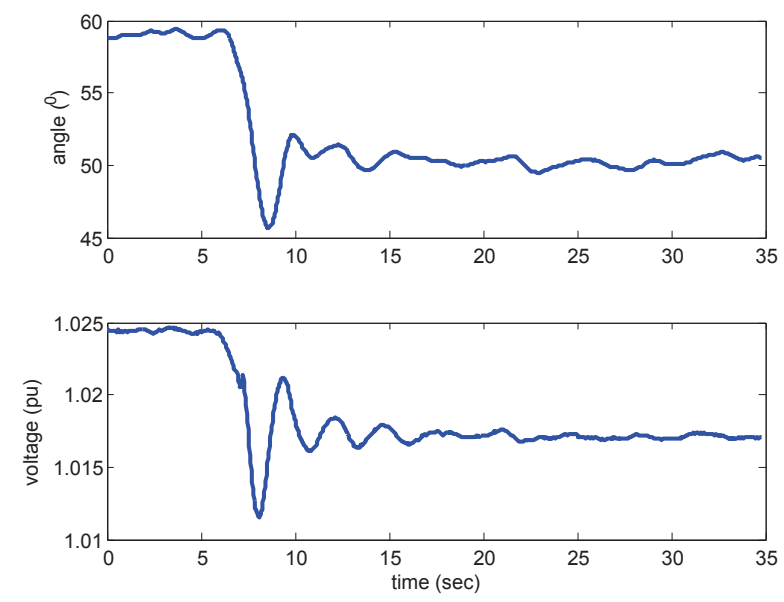

Fig. 7. Voltage phase angle and magnitudes at $P_{0}$.

The voltage magnitudes for the three buses are plotted in Fig. 9. From Fig. 9, the voltage oscillation components and the steady-state voltage components at one instant can be found. The values listed as follows correspond to 12.3 seconds.

\begin{tabular}{|c|c|c|}
\hline$V_{3 s s}=1.017$ & $V_{3 n}=0.0014$ & 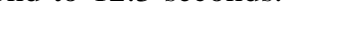 \\
\hline$V_{101 s s}=1.004$ & $V_{101 n}=0.0030$ & where $V_{3 s s}$ is the steady \\
\hline$V_{13 s s}=0.998$ & $V_{13 n}=0.0039$ & \\
\hline
\end{tabular}



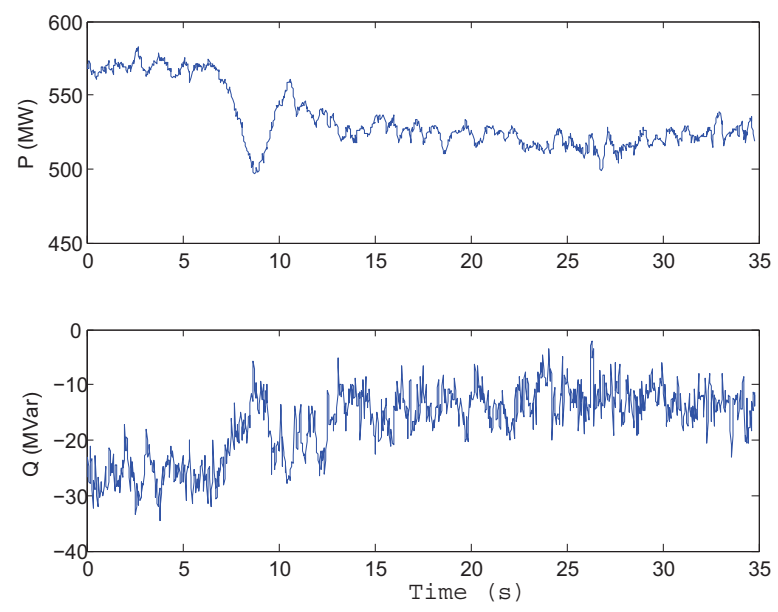

Fig. 8. Power flower from $P_{3}-P_{4}$.

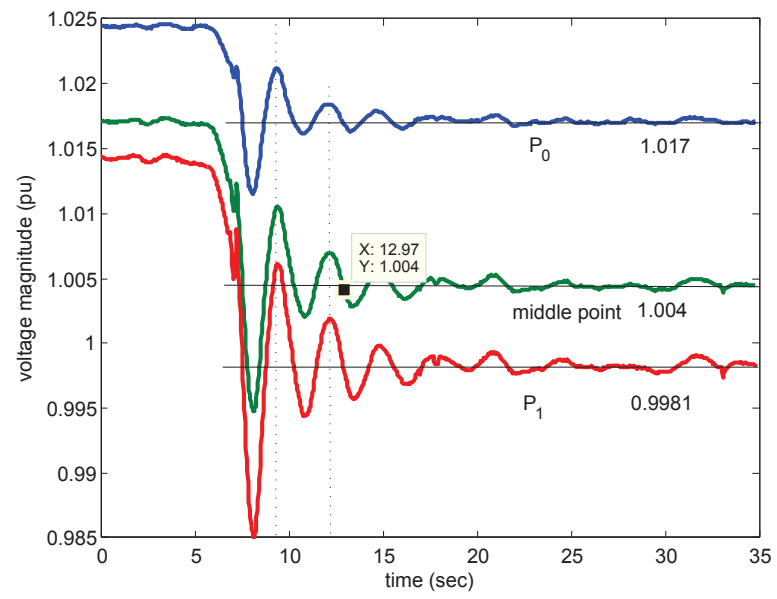

Fig. 9. Voltage magnitudes for the three buses.

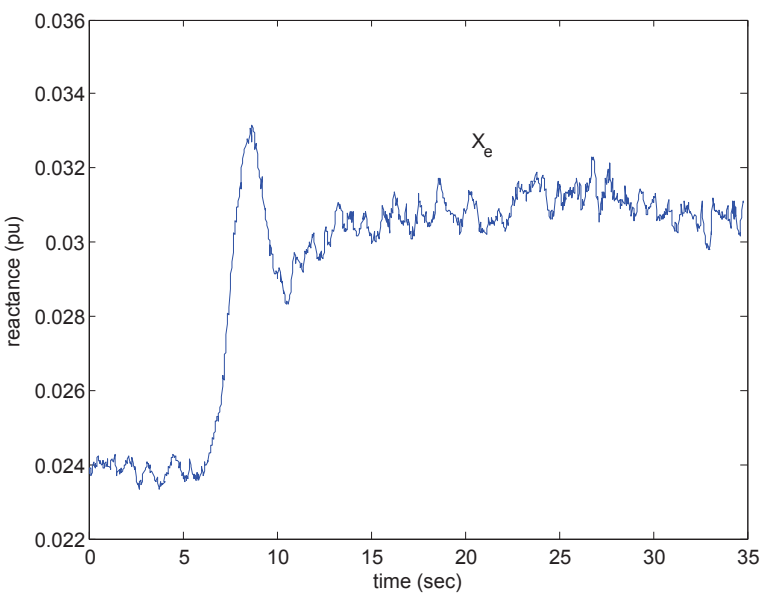

Fig. 10. Transfer path reactance. state value of the receiving end bus voltage $V_{13}, V_{101}$ is the bus at the middle point and $V_{101 s s}$ is its steady state value.

The transfer path reactance is computed from the voltage phasors and the current phasor. PMU data give real power and reactive power. Current phasors can be computed from the real power, reactive power and the voltage phasor. The reactance is shown in Fig. 10. A mean value is chosen for $x_{e}$ and the value is $0.0307 \mathrm{pu}$.

With the three voltage oscillation components and the transfer path reactance available, equivalent generator internal transient reactances can be found by solving two equations (1) and (2): $x_{1}^{\prime}=0.0099$ and $x_{2}^{\prime}=0.0647$.

The internal generator voltages can then be computed from the measured voltage, current and estimated reactances. Fig. 11 presents the measured voltage and the estimated generator internal voltages. The solid lines denote the measurements while the dotted lines denote the estimation. Fig. 12 presents the estimated internal voltage angles and the measurements. These angles are all related to the voltage phase angle at $P_{1}$.

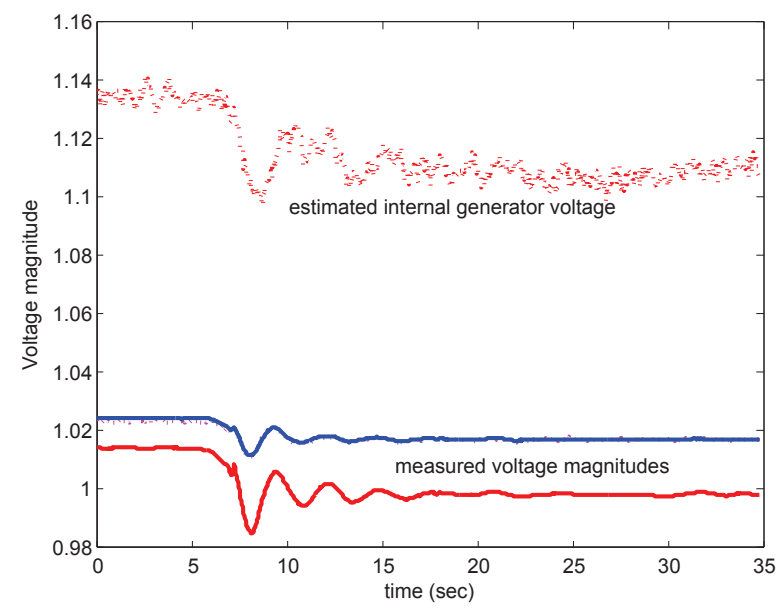

Fig. 11. Measurements and the estimated internal generator voltages.

The rotor speed deviations can also be estimated by the PMU data. The two rotor speed deviations should have op-

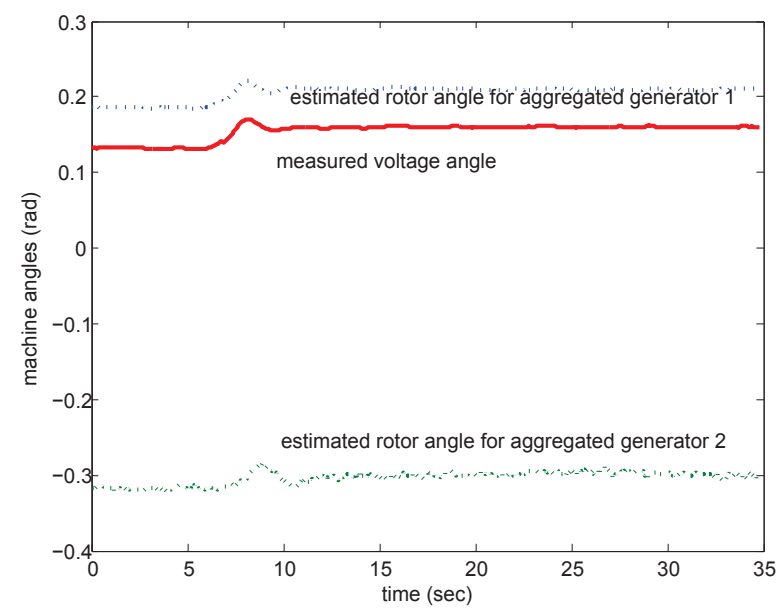

Fig. 12. Measured voltage phase angles and the estimated internal generator angles. 
posite polarity for $H_{1}$ and $H_{2}$ to have reasonable values [1]. Since this event involves frequency drop, the rotor speed deviations are always negative. This can lead to a negative inertia which is not reasonable. The solution for the real-world case is to use relative rotor speeds instead of absolute rotor speeds. In this case, $P_{1}$ is chosen as the reference bus. All phase angles are computed based on the voltage phase angle at $P_{1}$. The estimated rotor speed deviations will then have a base value zero as shown in Fig. 13.

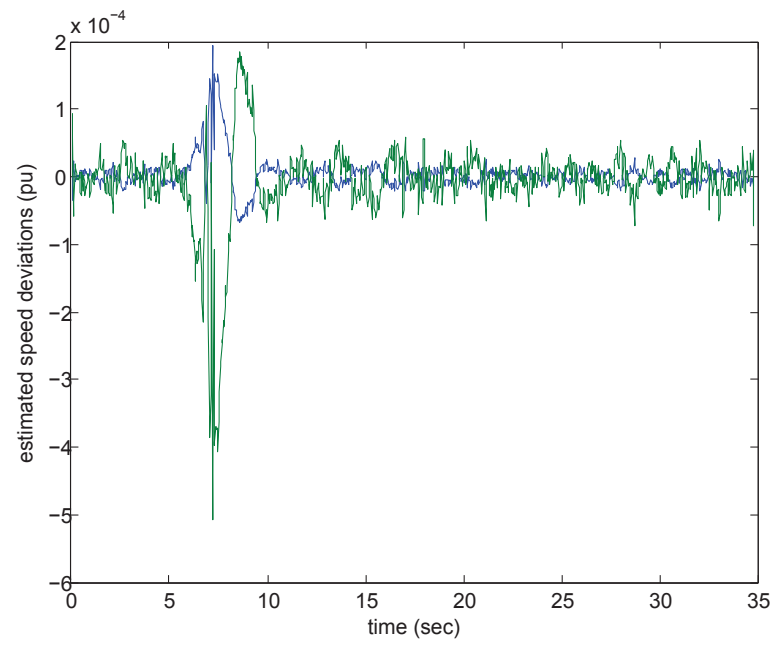

Fig. 13. Estimated rotor speed deviations.

Finally, based on the rotor speed deviation estimation [1] and the aggregated inertia, the inertia for each equivalent generator can be estimated. The inertias are shown in Fig. 14. The aggregated inertia is about 350 s.pu. The inertia constant for the area behind $P_{0}$ is about 1200 s.pu, while the inertia constant for the area behind $P_{1}$ is about 500 s.pu.

\section{B. Finite Difference Method}

The method proposed in [12] is applied in this subsection to the PMU data. We will apply the algorithm to the PMU data from $P_{0}$. The inputs of the estimation algorithm are

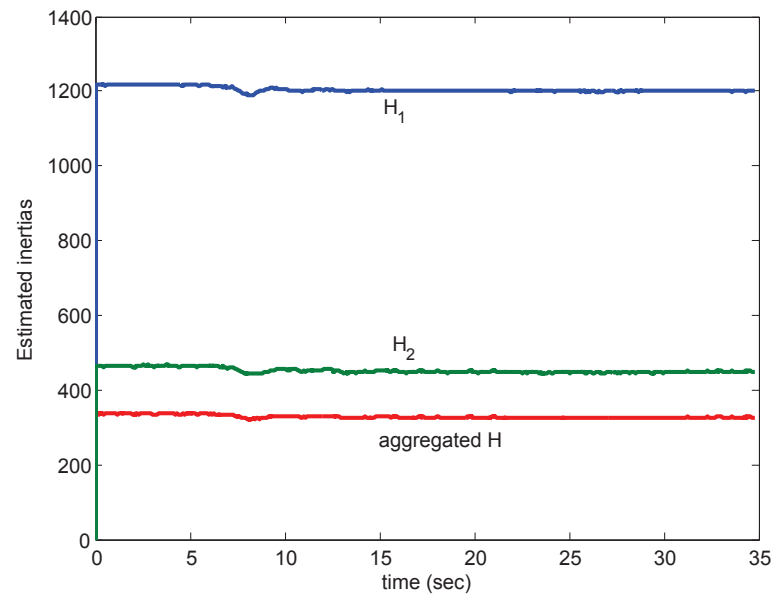

Fig. 14. Estimated inertia constants. a time series of voltage phasors (angle and magnitude) and current phasor as shown in Fig. 15. The phase angles are all related to the voltage phase angle at bus $P_{1}$. The outputs of the estimation algorithm are internal generator voltage phasor (angle and magnitude), transient reactance and equivalent inertia.
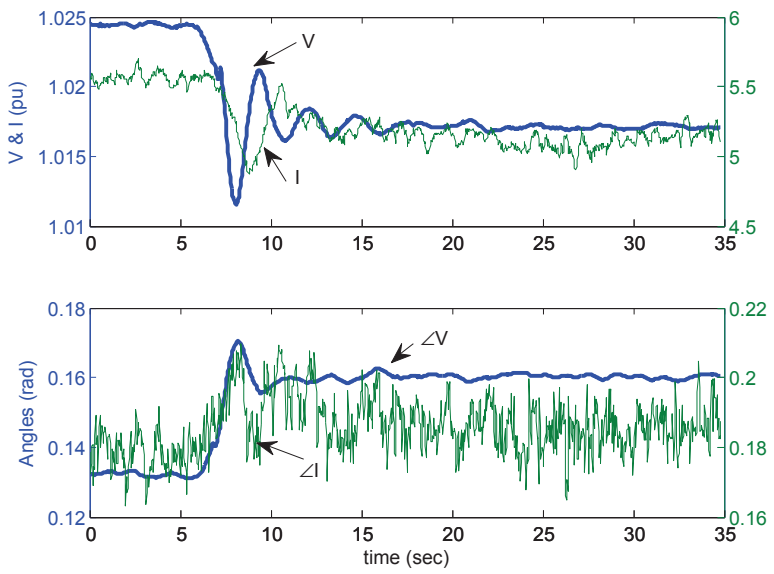

Fig. 15. Inputs of the estimation algorithm: Finite Difference Method. Phase angles are relative to the ones of $P_{1}$.

The first step of the finite difference method is to estimate the transient reactance by curve fitting (6). We find that $x_{d}^{\prime}=$ 0.0209 .

With $x_{d}^{\prime}$ available, the internal voltage phasor $\bar{E}$ can be estimated. Figs. 16 and 17 present the internal voltage magnitude and the phase angle. The measured voltage magnitude and phase angle are also presented in the figures.

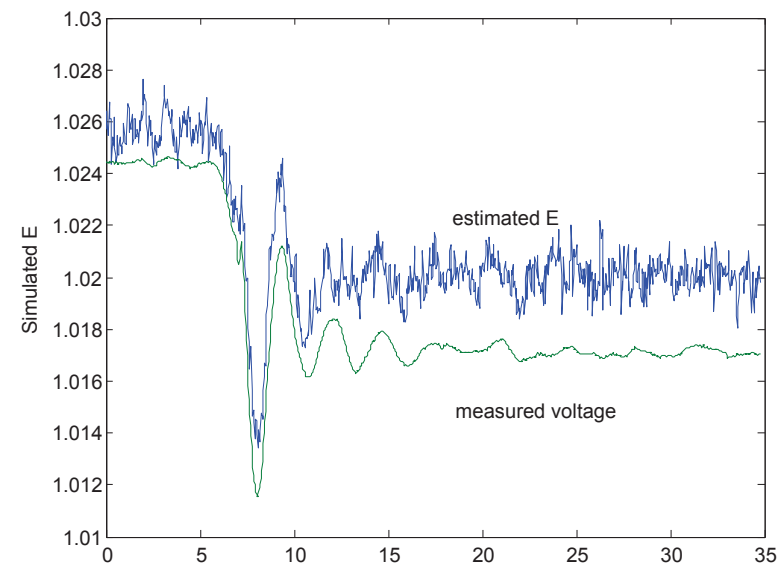

Fig. 16. Estimated internal voltage magnitude compared with the measured voltage.

In order to obtain the equivalent inertia, the derivative of $P_{e}$ and the 3rd derivative of $\delta$ should be obtained. It is found that the derivatives of $\delta$ have white noise which is difficult to be filtered out as shown in Fig. 18. With white noise presented, the computed inertia also presents significant white noises. White noise is the key issue that affects the accuracy of this algorithm.

To solve this issue, digital filter techniques is introduced to deal with the estimated rotor angle. Chebyshev filter is used 


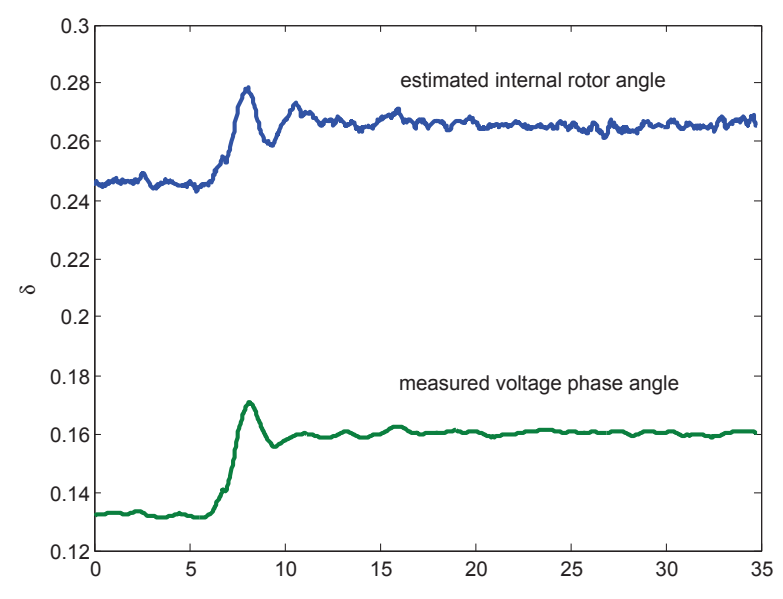

Fig. 17. Estimated internal voltage phase angle compared with the simulated rotor angle.
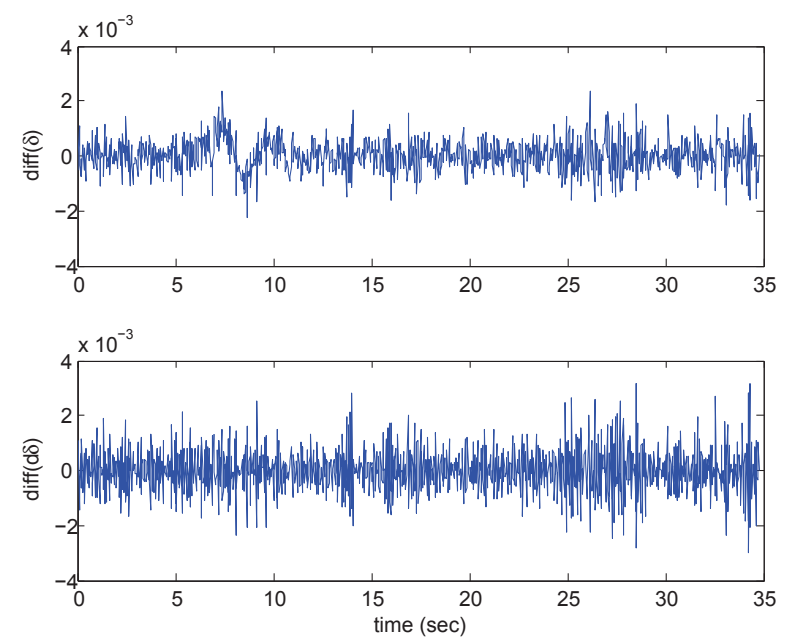

Fig. 18. Derivatives with white noise.

in this case with the sampling window set to be 64 samples to carry out the filtering process. The Chebyshev filter timedomain and frequency domain function are shown in Fig. 19. With Chebyshev filter applied, the internal angle waveform will be smoothed out (shown in Fig. 20). Similarly, the power waveform is smoothed out (shown in Fig. 21). The derivatives after applying digital filtering technique are shown in Fig. 22. Compared to noise polluted waveforms shown in Fig. 18, the waveforms after Chebyshev filter are much smoother.

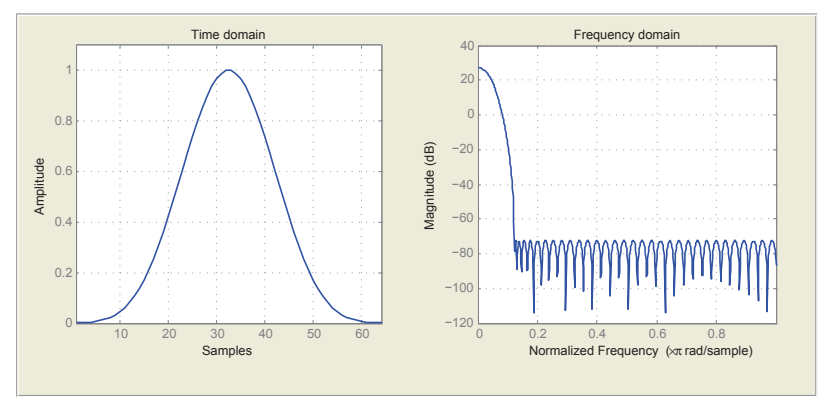

Fig. 19. Chebyshev filter.

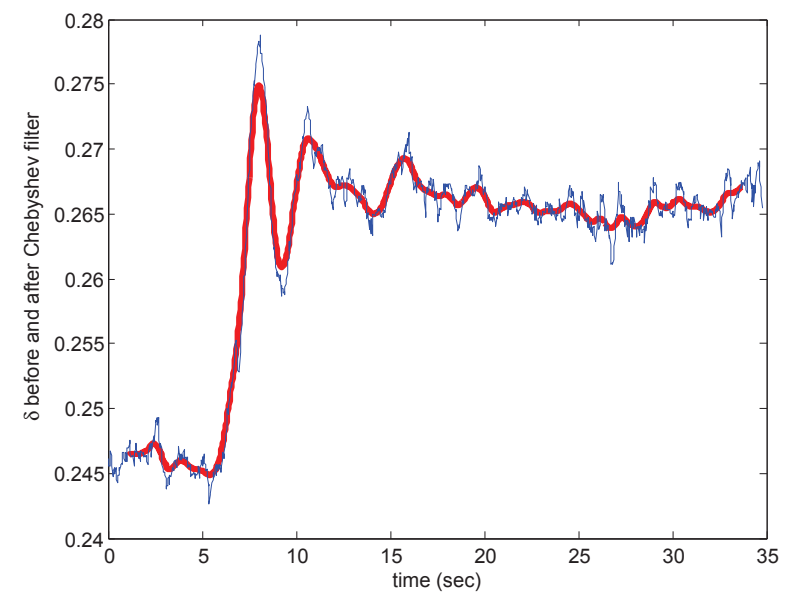

Fig. 20. $\delta$ before and after Chebyshev filter.

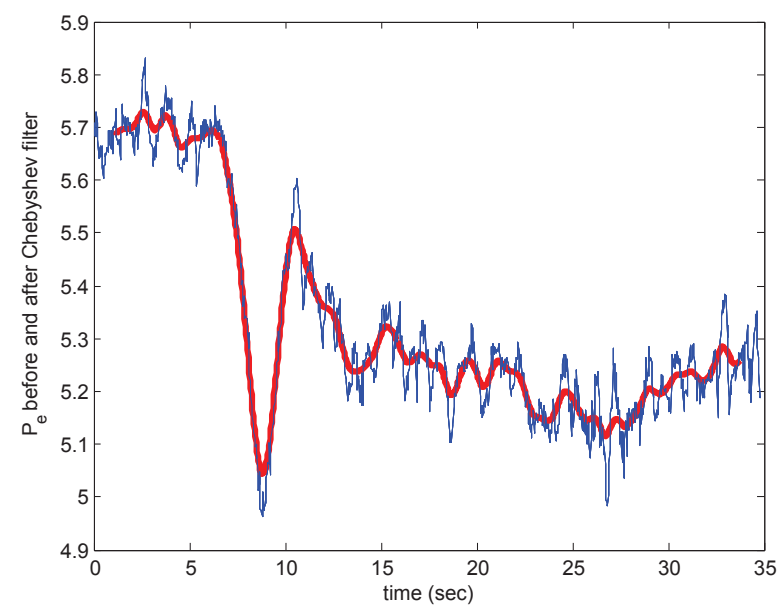

Fig. 21. $P_{e}$ before and after Chebyshev filter.
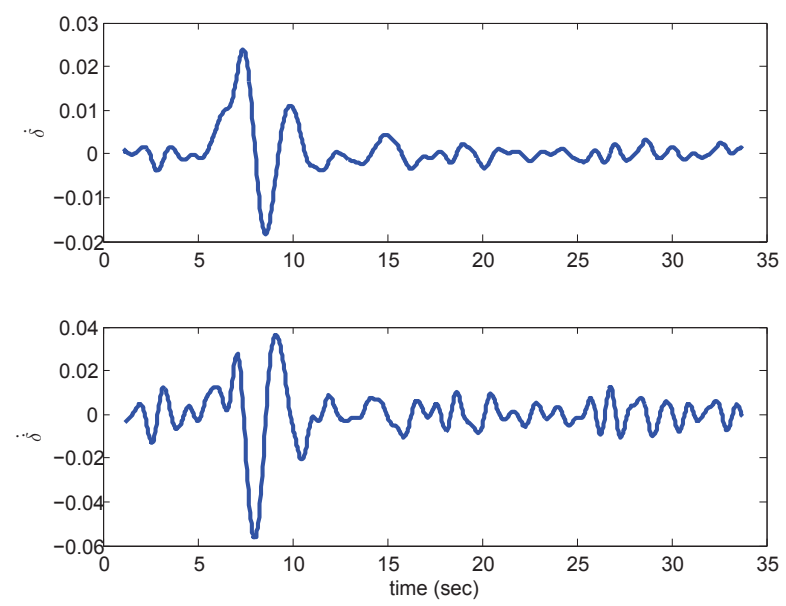

Fig. 22. Derivatives of $\delta$ after Chebyshev filter. 


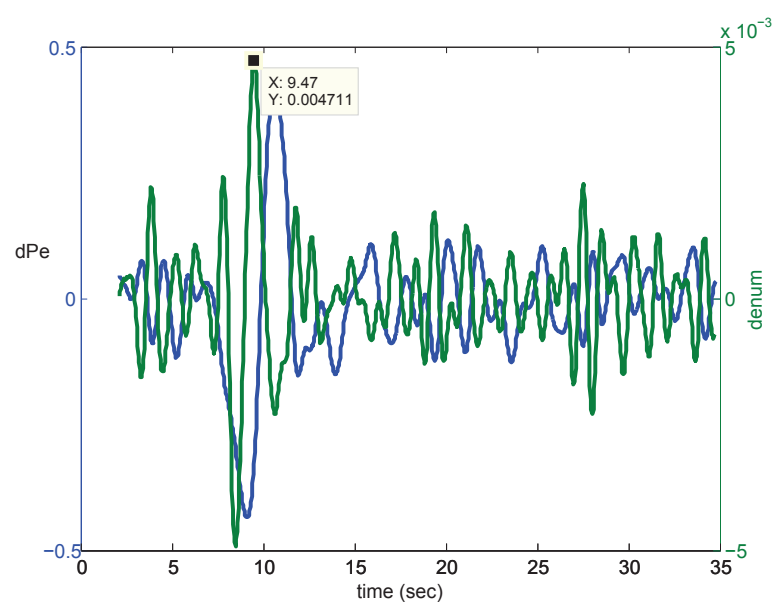

Fig. 23. Nominator and denominator for inertia computation.

With the smoothed waveforms, the first order derivative of $P_{e}$ and the 3 rd order derivative of $\delta$ are obtained and presented in Fig. 23. The equivalent system inertia can be computed from Equation (10) which is repeated here:

$$
H=-\frac{\Omega}{2}\left(\frac{\partial P_{e}}{\partial t}\right) /\left(\frac{\partial^{3} \delta}{\partial t^{3}}\right)
$$

Fig. 23 presents the derivative of $P_{e}$ and the denominator in (10). We select an instant at $t=9.47$ second and find the $\frac{d P_{e}}{d t}$ and $\frac{d^{3} \delta}{d t}$. The inertia for the equivalent generator behind $P_{0}$ can be found to be 1300 seconds pu.

\section{Discussion}

One of the key difference of the two methods reside in the assumption of system. The first method assumes a two-area system with a radial path. The second method assumes a single machine connected to an infinity bus. In the first method, two PMU locations are identified as the terminal buses for the two areas. However, the real system is a complex interconnected system and it does not fit the assumption of a two-area radial system very well. Therefore reactance estimation could deviate from the true value. Due to the reactance estimation deviation, the estimation of inertia can also be influenced. In the second example, a PMU location is selected as the terminal bus from an equivalent generator. This generator is connected to the big system (infinity bus) through a line. Such assumption is more reasonable for the real system. The validation of the estimation results should be conducted for simulation models very similar to the assumption. Validation of Chow's method has been done in [1] Validation for the finite difference based method has been done in the authors paper [12].

\section{CONCLUding Remarks}

In this paper, estimation algorithms are applied to the RTDMS data for a real-world generator trip event. four challenges are tackled and they are:

- Obtain suitable RTDMS data which reflect system angular oscillations. This proves to be the most difficult task for the project. In the majority of the event data, obvious angular oscillations are difficult to find. The event data presented in this paper are suitable for the study.

- With data available, the second challenging task is to identify the transfer path and identify which PMUs to be used for estimation algorithms. If the PMU location is chosen randomly and the two PMUs cannot reflect a radial path, the results make little sense.

- For the algorithm of using two PMUs to estimate a transfer path (Chow's method), one challenge is to compute $H_{1}$ and $H_{2}$ from the aggregated inertia based on the estimated rotor speed deviations $(\Delta \omega)$ of the two equivalent generators. The two rotor speed deviations should have opposite polarity for $H_{1}$ and $H_{2}$ to have reasonable values. Since this event involves frequency drop, the rotor speed deviations are always negative. This can lead to a negative inertia which is not reasonable.

The solution for the real-world case is to use relative rotor speeds instead of absolute rotor speeds. One bus in the transfer path will be made as the reference bus. All phase angles are computed based on this reference voltage.

- For the finite difference method estimate an equivalent generator for one area, the key challenge is the white noise presented in the signal waveforms. This white noise can damage the waveforms of the derivatives of the signal and the results are meaningless.

A solution is to use Chebyshev digital filter to get rid of the white noise for the initial signals and smooth the waveforms. The resulting derivatives are smooth. This method can then be used to compute estimated inertia.

The estimation results from both algorithms are compared. The estimated reactance from Chow's method is $0.01 \mathrm{pu}$ while the one from finite difference method is $0.02 \mathrm{pu}$. The estimated inertia for the equivalent generator behind $P_{0}$ is $1200 \mathrm{s.pu}$ based on Chow's method and 1300 s.pu based on the finite difference method. The two resulting inertias have less than $10 \%$ of difference. Assuming that the inertia for one 1000 MW generator is 5 seconds, Area 1 is equivalent to having 241000 MW generators. From Chow's method, the inertia for Area 2 is about 500 s.pu, which is equivalent of ten 1000 MW generators.

\section{ACKNOLOWLEDGEMENT}

The authors would like to acknowledge the help from Midwest ISO (Terry Bilke and Anna Zwergel) for providing RTDMS data and help in real-world data analysis. Prof. Gokhan Mumcu at USF is acknowledged for his help in providing insights in digital filter techniques.

\section{REFERENCES}

[1] J. H. Chow, A. C. Chakrabortty, L. Vanfretti, and M. Arcak, "Estimation of radial power system transfer path dynamic parameters using synchronized phasor data," IEEE Trans. Power Syst., vol. 23, no. 2, pp. 564-571, May 2008.

[2] A. Agarwal, J. Ballance, B. Bhargava, J. Dyer, K. Martin, and J. Mo, "Real time dynamics monitoring system (rtdms) for use with synchrophasor technology in power systems," Proc. of IEEE Power \& Energy General Meeting, July 2011.

[3] J. Melgoza, G. Heydt, A. Keyhani, B. Agrawal, and D. Selin, "Synchronous machine parameter estimation using the hartley series," IEEE Trans. Energy Convers., vol. 16, no. 1, pp. 49-54, mar 2001. 
[4] E. Kyriakides, G. Heydt, and V. Vittal, "Online parameter estimation of round rotor synchronous generators including magnetic saturation," IEEE Trans. Energy Convers., vol. 20, no. 3, pp. 529-537, sep 2005.

[5] J. Melgoza, G. Heydt, A. Keyhani, B. Agrawal, and D. Selin, "An algebraic approach for identifying operating point dependent parameters of synchronous machines using orthogonal series expansions," IEEE Trans. Energy Convers., vol. 16, no. 1, pp. 92-98, mar 2001.

[6] E. Kyriakides, G. Heydt, and V. Vittal, "On-line estimation of synchronous generator parameters using a damper current observer and a graphic user interface," IEEE Trans. Energy Convers., vol. 19, no. 3, pp. 499-507, sep 2004.

[7] J. Chang, G. Taranto, and J. Chow, "Dynamic state estimation in power system using a gain-scheduled nonlinear observer," Proc. of the 4th IEEE Conference on Control Applications, pp. 221-226, sep 1995.

[8] Z. Huang, K. Schneider, and J. Nieplocha, "Feasibility studies of applying kalman filter techniques to power system dynamic state estimation," Proc. of International Power Engineering Conference (IPEC), pp. 376382, Dec 2007.

[9] Z. Huang, P. Du, D. Losterev, and B. Yang, "Application of extended kalman filter techniques for dynamic model parameter calibration," Proc. of IEEE Power \& Energy General Meeting, July 2009.

[10] K. Kalsi, Y. Sun, Z. Huang, P. Du, R. diao, K. Anderson, Y. Li, and B. Lee, "Calibrating multi-machine power system parameters with the Extended Kalman Filter," Proc. of IEEE Power \& Energy General Meeting, July 2011.

[11] Y. Wehbe and L. Fan, "Estimation of a shunted radial transfer path dynamics using pmus," IEEE PES General Meeting, Jul. 2011.

[12] _ - "Estimating synchronous machine parameters with pmu measurements," submitted to IEEE Energy Conversio Congress and Exhibition 2012.

[13] I. Kamwa and R. Grondin, "Fast adaptive schemes for tracking voltage phasor and local frequency in power transmission and distribution systems," IEEE Trans. Power Del., vol. 7, no. 2, pp. 789-795, apr 1992.

Lingling Fan (S'99-M'02-SM'08) is an assistant professor in University of South Florida (Tampa, FL) since Aug. 2009. She received the BS, MS degrees in electrical engineering from Southeast University, Nanjing, China, in 1994 and 1997, respectively. She received Ph.D. degree in electrical engineering from West Virginia University in 2001. She was a senior engineer in transmission asset management department in Midwest ISO, St. Paul, Minnesota (2001-2007) and an assistant professor in North Dakota State University (2007-2009). Her research interests include modeling and control of energy systems, large-scale power systems planning and operation.

Zhixin Miao (S'00-M'03-SM'09) is with University of South Florida since Aug. 2009. He received his BSEE from Huazhong University of Science \& Technology, Wuhan, China, in 1992. He received his MSEE from the graduate school of Nanjing Automation Research Institute in 1997 and Ph.D. in Electrical Engineering from West Virginia University in 2002. He was with transmission asset management department in Midwest ISO, St. Paul, Minnesota from 2002 to Aug. 2009. His research interests include dynamics modeling of electric machinery and power system, power system protection, reliability and economics.

Yasser Wehbe (S'10) is currently a Ph.D. candidate at University of South Florida. His research areas focus on system identification applications in power systems. 